\title{
Total Body Water and Failure to Control Blood Pressure by Medication in Hemodialysis Patients
}

\author{
Patrícia Santi Xavier Bárbara Perez Vogt Luis Cuadrado Martin \\ Francieli Vaninni Aline Araújo Antunes Daniela Ponce \\ Jacqueline Costa Teixeira Caramori Rosana dos Santos e Silva Martin \\ Roberto Jorge da Silva Franco Pasqual Barretti \\ Botucatu School of Medicine, São Paulo State University, São Paulo, Brazil
}

\author{
Key Words \\ Extracellular volume $\cdot$ Bioimpedance analysis $\cdot$ Blood pressure $\cdot$ Hemodialysis
}

\begin{abstract}
Background: Volume overload is the main factor responsible for the pathogenesis of hypertension in dialysis patients. Few studies have evaluated the interpretation of the parameters obtained by bioelectrical impedance (BIA) to manage these patients. The aim of this study was to assess the best cutoff level of volume overload obtained by BIA able to predict the absence of hypertension control in hemodialysis patients. Methods: Volume overload was calculated as the difference between total body water (TBW) measured by bioimpedance and TBW estimated by the Watson formula in chronic stable hemodialysis patients. Inadequate control of blood pressure (BP) was defined as the mean of measurements obtained before five hemodialysis sessions $\geq 140 \times 90 \mathrm{~mm} \mathrm{Hg}$. The best cutoff level of volume overload assessed by BIA able to predict the absence of BP control in patients on chronic hemodialysis was determined by the receiver operating characteristic (ROC) curve using the Youden method. Results: We included 205 patients, 53\% male, aged $56 \pm 14.5$ years. The largest area under the ROC curve was found for predialysis volume overload $(0.660,95 \%$ CI $0.556-0.765, p=$ $0.004)$. The ROC curve of postdialysis volume overload also reaches statistical significance. The best cutoff point was found for predialysis volume overload $\geq 1.4$ liters with a sensitivity of $69 \%$ and a specificity of $67 \%$. Conclusion: The association of TBW and inadequate BP control highlights the importance of volume management in hemodialysis patients. Predialysis volume overload of 1.4 liters was the parameter that best discriminated the presence of inadequate BP control.


Santi Xavier et al.: Total Body Water and Failure to Control Blood Pressure by Medication in Hemodialysis Patients

\section{Introduction}

Arterial hypertension has a high prevalence in dialysis patients ranging from 60 to $80 \%$ [1]. A study carried out in our clinic showed that $66 \%$ of patients on regular hemodialysis (HD) had inadequate blood pressure (BP) control [2], and this value reached approximately $86 \%$ when added to those who had BP controlled by antihypertensives (the prevalence being similar in the literature [3]). The available literature considers volume overload as the main factor responsible for the pathogenesis of arterial hypertension in dialysis patients [4]; however, some studies have reported a nonlinear relation between the BP levels and interdialytic weight gain (IDWG). Savage et al. [5] reported no correlation between interdialytic BP change, evaluated by 48-hour ambulatory BP monitoring, and weight gain in HD patients and a Brazilian study did not find a significant correlation between the average 44-hour BP and IDWG [6]. Thus, IDWG cannot be considered as a single parameter for volume overload quantification in these patients, and it is fundamental for the clinical practice to seek other methods for this evaluation.

Bioelectrical impedance (BIA) is a simple and low-cost method that can be useful in clinical practice to estimate the volume overload in HD patients [7-9]. However, until the present time, few studies showed parameters for the interpretation of the data provided by BIA associating them with total body water (TBW) or BP control.

Katzarski et al. [10], using multifrequency BIA, have validated a parameter for the evaluation of the hydration state in HD; however, studies using monofrequency BIA with this objective have not been published. This study aimed to evaluate the best cutoff point of the TBW assessed by monofrequency BIA, which is able to predict the lack of BP control in HD patients.

\section{Patients and Methods}

This retrospective cross-sectional study was performed at the Dialysis Unit of the University Hospital of the Botucatu Medical School, UNESP, Brazil. All patients having been treated for at least 3 months with regular HD were included (the information was obtained from the medical records). The study was approved by the institutional ethics committee. Exclusion criteria were patients with a heart failure, a liver failure, alcoholism, or an artificial pacemaker. Predialysis BP was defined as the average of the measurements obtained at the beginning of five consecutive dialysis sessions preceding the assessment by BIA. Body weight was evaluated during two periods, before and after dialysis, over the last five sessions that preceded the BIA. The mean IDWG was obtained by subtracting the average of the postdialysis weight of the last five sessions from the average of predialysis weights.

Demographic and clinical data as well as drug prescriptions were obtained from medical records. Inadequate BP control was defined as BP $\geq 140 \times 90 \mathrm{~mm} \mathrm{Hg}$ in the predialysis period.

To obtain the parameters of body water volume, a monofrequency BIA device (Biodynamics ${ }^{\circledR}$, model $450,800 \mu \mathrm{A}, 50 \mathrm{kHz}$ ) was used which measures the whole body impedance. This assessment was performed $30 \mathrm{~min}$ after the end of the dialysis session. The measurements were performed with the patient in the supine position, on the side opposite the HD vascular access. An electrode was attached to the dorsal surface of the wrist and another on the dorsal surface of the third metacarpal bone. The second pair of electrodes was placed on the anterior surface of the ankle and the third metatarsal bone, respectively $[11,12]$. The estimated parameters by BIA included extracellular water (ECW), intracellular water and TBW. The ratio between ECW and TBW (ECW/TBW ratio) was calculated. The TBW value obtained by BIA was compared to the value estimated by the Watson formula [13]; the difference 
Table 1. Clinical and demographic characteristics of the sample studied
Santi Xavier et al.: Total Body Water and Failure to Control Blood Pressure by Medication in Hemodialysis Patients

\begin{tabular}{ll}
\hline Characteristic & Value \\
\hline Mean age \pm SD, years & $56 \pm 14.5$ \\
Female gender, \% & 47 \\
Race, \% & \\
$\quad$ White & 69 \\
$\quad$ Black & 16 \\
$\quad$ Mestizo & 14 \\
$\quad$ Asian & 1 \\
Diabetics, \% & 37 \\
Antihypertensive drug classes, n (\%) & $2(1-3)$ \\
Mean systolic BP \pm SD, mm Hg & $141 \pm 16.8$ \\
Mean diastolic BP \pm SD, mm Hg & $86 \pm 14.4$ \\
Mean body mass index \pm SD & $25.0 \pm 5.19$ \\
\hline
\end{tabular}

between them was considered as postdialysis fluid overload. Predialysis fluid overload was defined as the sum of postdialysis fluid overload plus the mean IDWG.

\section{Statistical Analysis}

Data were expressed as means \pm standard deviations or medians and first and third quartiles, according to the variable distribution. For the comparison of several groups, one-way analysis of variance followed by Tukey's test was used. Receiver operating characteristic (ROC) curves were plotted, considering as endpoint variable the presence of inadequate predialysis BP control and as independent variables the presence of pre- and postdialysis fluid overload and the ECW/TBW ratio. The best cutoff point was determined by the Youden index (higher sum of sensitivity plus specificity). A p value $<0.05$ was considered statistically significant.

\section{Results}

We enrolled 205 patients, $53 \%$ of whom were male. Their mean age was $56 \pm 14.5$ years. The median number of antihypertensive classes per patient was 2 (interquartile range 1-3). The remaining characteristics are shown in table 1.

Patients were divided into two groups according to BP control classification (adequate or inadequate). Table 2 shows the number of prescribed antihypertensive classes and the measurements of pre- and postdialysis fluid overload in these groups. There was no patient taking 4 or more classes of antihypertensive drugs among those with adequate BP control. Fluid overload was higher among patients with inadequate BP control taking 4 or more classes of antihypertensive drugs compared to those with adequate BP control and receiving 1 class of antihypertensive drugs $(p=0.02)$. Figure 1 shows the areas under the ROC curve of the measures of pre- and postdialysis fluid overload and the ECW/TBW ratio to predict the presence of inadequate BP control. The greatest area under the ROC curve corresponds to predialysis fluid overload $(0.660,95 \%$ CI $0.556-0.765, \mathrm{p}=0.004)$; the ROC curve of postdialysis fluid overload also reached statistical significance $(0.617,95 \%$ CI $0.509-0.724, p=$ 0.039 ). The best cutoff point was a predialysis fluid overload of 1.4 liters with a sensitivity of $69 \%$ and a specificity of $67 \%$. The ECW/TBW ratio did not have a significant association with inadequate BP control. 
Fig. 1. ROC curves: pre- and postdialysis fluid overload, ECW/ TBW ratio and the presence of inadequate BP control.

Table 2. Pre- and postdialysis fluid overload, according to the number of antihypertensive drugs taken by patients, with adequate or inadequate BP control

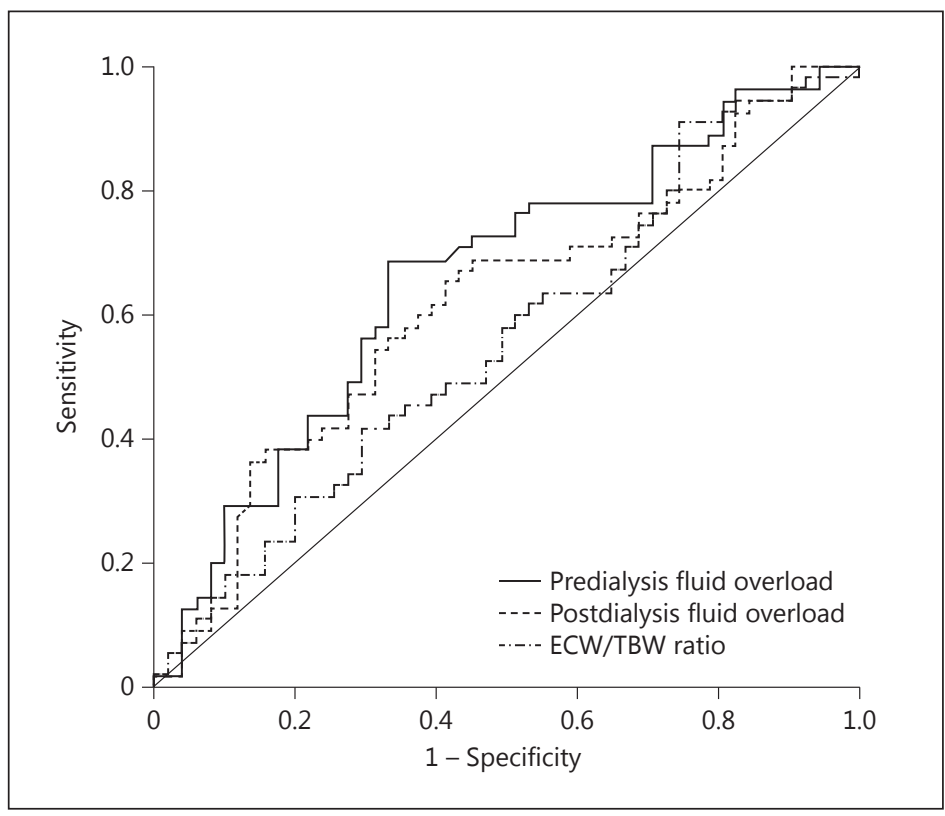

\begin{tabular}{lll}
\hline $\begin{array}{l}\text { Number of } \\
\text { classes }\end{array}$ & $\begin{array}{l}\text { Predialysis fluid } \\
\text { overload, } 1\end{array}$ & $\begin{array}{l}\text { Postdialysis fluid } \\
\text { overload, } 1\end{array}$ \\
\hline $\begin{array}{l}\text { Adequate } \\
0\end{array}$ & $0.42(-0.86 ; 2.91)$ & $-1.89(-3.24 ; 0.68)$ \\
1 & $-0.63(-2.65 ; 1.26)$ & $-1.96(-5.71 ;-0.62)$ \\
2 & $0.08(-3.03 ; 1.88)$ & $-2.03(-4.67 ;-0.89)$ \\
3 & $0.91(-1.42 ; 5.49)$ & $-0.49(-2.74 ; 2.90)$ \\
Inadequate & & \\
0 & $0.53(-2.36 ; 1.81)$ & $-1.70(-4.14 ; 0.54)$ \\
1 & $0.81(-1.10 ; 3.37)$ & $-1.65(-4.38 ; 1.07)$ \\
2 & $2.34(-0.85 ; 3.94)$ & $-0.49(-3.36 ; 1.26)$ \\
3 & $1.96(-0.61 ; 3.40)$ & $-0.87(-3.53 ; 1.00)$ \\
4 & $3.09(1.49 ; 6.33)^{\mathrm{b}}$ & $-0.32(-1.29 ; 2.66)^{\mathrm{a}}$ \\
\hline
\end{tabular}

Figures in parentheses indicate interquartile interval.

${ }^{\mathrm{a}} \mathrm{p}=0.02$ vs. adequate using 1 class of antihypertensive drugs; ${ }^{\mathrm{b}} \mathrm{p}=$ 0.02 vs. adequate using 1 class of antihypertensive drugs.

\section{Discussion}

BIA is a noninvasive and nonexpensive method with a high sensitivity and reproducibility [14]. In this study, TBW measured by BIA was associated with BP control in HD patients. For instance, fluid overload estimated by the difference between TBW and body water volume calculated by an anthropometric formula was able to predict the lack of BP control in spite of medication taken by these patients, and predialysis fluid overload was a valid parameter to predict it.

We used the Watson formula [13] to estimate TBW, an equation validated by a tracer dilution technique in normal people to estimate adequate fluid volume and supply a reference value regarding volume overload in comparison with measured TBW. 
Santi Xavier et al.: Total Body Water and Failure to Control Blood Pressure by Medication in Hemodialysis Patients

Predialysis volume overload was utilized considering this moment as the volume overload peak; thus, predialysis volume overload was estimated adding IDWG to postdialysis volume overload, directly measured by BIA.

To estimate BP control, the average of five sessions was used, because in a previous study we verified that this number of measurements is best to estimate ambulatory BP, as well as left ventricular mass [15]. In addition to BP evaluation, we used the average of five sessions to estimate IDWG.

Another study using monofrequency BIA showed that hypertensive patients had a higher percentage of ECW; in contrast, the present study found no differences in TBW between normotensive and hypertensive patients. Some studies have suggested parameters of BIA as tools to evaluate the hydration status in patients with chronic kidney disease [17-19]; however, none of them showed a cutoff point applicable to clinical practice predicting the lack of BP control particularly using monofrequency BIA. We used monofrequency BIA which has advantages in terms of handling simplicity and low cost to facilitate the use in developing countries [16].

Other methods to evaluate fluid overload include biochemical markers, such as atrial natriuretic peptide and guanidine cyclic monophosphate; these are high-sensitivity methods but with specific limitations, for instance congestive heart failure, mitral and tricuspid valve diseases, and atrial hemodynamic changes. Furthermore, these markers require an advanced technology at high costs, available to only a few centers, which limits its use for clinical routine.

Some limitations of this study should be taken into account; in particular, multifrequency BIA is more accurate to measure ECW and intracellular water than monofrequency BIA [17, 20-22], which we used; this may explain the absence of a relationship between ECW/TBW ratio and $\mathrm{BP}$ control. In addition, BIA measurements were not performed before dialysis, the study design was cross-sectional, and its results need confirmation by a longitudinal study. The sensitivity of $69 \%$ and specificity of $67 \%$ was not perfect, but significant. So, the clinical use of this parameter is possible; however, we need to interpret the results cautiously and also consider other clinical parameters of dry weight.

On the other hand, strengths of this study should also be highlighted. This work is the first to establish a simple parameter for the interpretation of data from monofrequency BIA regarding the evaluation of fluid overload, which allows establishing a practical reference for clinical practice. Indeed its results are supported by an expressive sample size reinforcing their consistency.

\section{Conclusion}

TBW assessment by BIA is associated with BP control in HD patients, reinforcing the importance of volume fluid management in these patients. Predialysis fluid overload was the best parameter to predict lack of BP control; it was possible to establish a cutoff point for use in daily clinical routine.

\section{References}

1 Rahman M, Dixit A, Donley V, Gupta S, Hanslik T, Lacson E, Oqundipe A, Weigel K, Smith MC: Factors associated with inadequate blood pressure control in hypertensive hemodialysis patients. Am J Kidney Dis 1999;33: 498-506.

2 Martin LC, Franco SJR: Renal disease as a cardiovascular risk factor. Arq Bras Cardiol 2005;85:432-436.

3 Salen MM, Bower J: Hypertension in the hemodialysis population: any relation to one year survival? Am J Kidney Dis 1996;28:737-740. 
Santi Xavier et al.: Total Body Water and Failure to Control Blood Pressure by Medication in Hemodialysis Patients

4 Hörl MP, Hörl WH: Hemodialysis-associated hypertension: pathophysiology and therapy. Am J Kidney Dis 2002;39:227-244.

5 Savage T, Fabbian F, Giles M, Tomson CR, Raine AE: Interdialytic weight gain and 48-h blood pressure in haemodialysis patients. Nephrol Dial Transplant 1997;12:2308-2311.

6 Santos SF, Mendes RB, Santos CA, Dorigo D, Peixoto AJ: Profile of interdialytic blood pressure in hemodialysis patients. Am J Nephrol 2003;23:96-105.

-7 Zhu F, Kotanko P, Handelman GJ, Raimann JG, Liu L, Carter M, Kuhlmann MK, Seibert E, Leonard EF, Levin NW: Estimation of normal hydration in dialysis patients using whole body and calf bioimpedance analysis. Physiol Meas 2011;32:887-902.

8 Kotanko P, Levin NW, Zhu F: Current state of bioimpedance technologies in dialysis. Nephrol Dial Transplant 2008;23:808-812.

9 Chen YC, Lin CJ, Wu CJ, Chen HH, Yeh JC: Comparison of extracellular volume and blood pressure in hemodialysis and peritoneal dialysis patients. Nephron Clin Pract 2009;113:c112-c116.

-10 Katzarski K, Charra B, Laurent G, Lopot F, Divino-Filho JC, Nisell J, Bergström J: Multifrequency bioimpedance in assessment of dry weight in haemodialysis. Nephrol Dial Transplant 1996;11(suppl 2):20-23.

11 van de Kerkhof J, Hermans M, Beerenhout C, Konings C, van der Sande FM, Kooman JP: Reference values for multifrequency bioimpedance analysis in dialysis patients. Blood Purif 2004;22:301-306.

12 Çelik G, Kara I, Yilmaz M, Apiliogullari S: The relationship between bioimpedance analysis, haemodynamic parameters of haemodialysis, biochemical parameters and dry weight. J Int Med Res 2011;39:2421-2428.

-13 Watson PE, Watson ID, Batt RD: Total body water volume for adult males and females estimated from simple anthropometric measurements. Am J Clin Nutr 1980;33:27-39.

14 Patel RV, Matthie JR, Withers PO, Peterson EL, Zarowitz BJ: Estimation of total body and extracellular water using single and multiple-frequency bioimpedance. Ann Pharmacother 1994;28:565-569.

15 Martin LC, Barretti P, Kochi AC, Balbi AL, Caramori JST, Franco RJS et al: Fisiopatologia da Hipertensão Arterial em Diálise. J Bras Nefrol 2006;28:225-231.

16 Lee SY, Gallagher D: Assessment methods in human body composition. Curr Opin Clin Nutr Metab Care 2008; 11:566-572.

17 Cha K, Chertow GM, Gonzalez J, Lazarus JM, Wilmore DW: Multifrequency bioelectrical impedance estimates the distribution of body water. J Appl Physiol 1995;79:1316-1319.

18 Fisch BJ, Spiegel DM: Assessment of excess fluid distribution in chronic hemodialysis patients using bioimpedance spectroscopy. Kidney Int 1996;49:1105-1109.

19 Charra B: Fluid balance, dry weight, and blood pressure in dialysis. Hemodial Int 2007;11:21-31.

20 Cornish BH, Thomas BJ, Ward LC: Improved prediction of extra cellular and total body water using impedance loci generated by multiple frequency bioelectric impedance analysis. Phys Med Biol 1993;38:337-346.

-21 van Marken Lichtenbelt WD, Westerterp KR, Wouters L, Luijendijk SC: Validation of bioelectrical-impedance measurements as a method to estimate body water compartments. Am J Clin Nutr 1994;60:159-166.

-22 Chanchairujira T, Mehta RL: Assessing fluid change in hemodialysis: whole body versus sum of segmental bioimpedance spectroscopy. Kidney Int 2001;60:2337-2342. 